\title{
Overall adjustment acupuncture for postmenopausal osteoporosis (PMOP): A study protocol for a randomized sham-controlled trial
}

ZQ ren

Nanjing University of Chinese Medicine

YF Wang

Yunnan University of Traditional Chinese Medicine

\section{GF Ao}

The first affilated hospital of Dali University

HX Chen

Yunnan University of Traditional Chinese Medicine

M Huang

Kunming Municipal Hospital of Traditional Chinese Medicine

MX Lai

Yunnan University of Traditional Chinese Medicine

HD Zhao

The first affiliated hospital of Dali University

R Zhao ( $\sim$ kmzhaorong@qq.com )

\section{Study protocol}

Keywords: Osteoporosis, Postmenopausal women, PMOP, acupuncture, sham acupuncture, Protocol

Posted Date: February 21st, 2020

DOI: https://doi.org/10.21203/rs.2.24185/v1

License: 두 (i) This work is licensed under a Creative Commons Attribution 4.0 International License. Read Full License

Version of Record: A version of this preprint was published at Trials on June 3rd, 2020. See the published version at https://doi.org/10.1186/s13063-020-04435-7. 


\section{Abstract}

Background: Osteoporosis is becoming more prevalent in aging societies worldwide, and the economic burden attributable to osteoporotic fractures is substantial. The medications presently available to treat osteoporosis have side effects, acupuncture is widely used for the postmenopausal women because of it is non-invasive and has fewer side effects, but the powerful evidence for the clinic is still insufficient. Our study intends to explore the effect of Overall adjustment acupuncture (OA) in the treatment of postmenopausal osteoporosis(PMOP).

Methods: This study is a randomized, sham-controlled, patient-and assessor-blinded trial and aims to evaluate the effect of OA in women with PMOP. We will recruit 104 women aged 45-70 years with a diagnosis of PMOP. Participants will be randomly allocated in a 1:1 ratio to the OA group and the sham acupuncture (SA) group. Both groups will receive real herbal medicine treatment as a basic treatment twice a day for three months, the OA group receive real acupuncture treatment and the SA group received placebo acupuncture treatment (non-penetrating,sham skin needle therapy, sham cupping). All patients will receive acupuncture treatment twice per week for three months. The primary outcome is bone mineral density(BMD) and secondary outcomes include estradiol(E2), follicle-stimulating hormone(FSH), bone gla protein(BGP), bone alkaline phosphatase (BALP), total antioxidant capacity(TAC), advanced oxidation protein products(AOPP) ,PPARy, $\beta$-Catenin,FoxO3a, visual pain scale score(VAS) , Traditional Chinese Medicine (TCM) syndrome scores and quality of daily life score(QOL). Outcome measures will be collected at baseline, middle of the treatment(1.5 months), the end of treatments(3month). The present protocol followed the SPIRIT guidelines and fulfilled the SPIRIT checklist.

Conclusion: This study will be conducted to compare the efficacy of OA versus SA. This trial will help to evaluate whether OA can effectively prevent and treat postmenopausal osteoporosis by improving the estrogen level of postmenopausal women, the mechanism is to improve the imbalance of osteogenic differentiation and lipogenesis of bone marrow cells under oxidative stress.

Trial registration: This trial was registed at Chinese Clinical Trial Registry, registration date: 5 August 2018.URL:http://www.chictr.org.cn,registration number:ChiCTR1800017581.

\section{Introduction}

The high incidence and the associated pain and fractures of postmenopausal osteoporosis( PMOP) have seriously threatened women's physical and mental health,as well as their life quality. According to statistics, about $1 / 4$ of perimenopausal women will develop osteoporosis, and women will lose bone mass rapidly within 5 years after menopause, the premenopausal loss is $2-3$ times than young women ${ }^{[1]}$. The prevention and treatment of PMOP is directly related to the physical and mental health of the most postmenopausal women, which has great social and practical significance.

Postmenopausal osteoporosis (PMOP) is caused by the decline of ovarian function, rapid aging of the body, decreased estrogen levels, hyperabsorptive function, and compensatory enhancement of osteoblast 
bone formation, all of these factors result in a high conversion of bone metabolism. Negative balance state eventually leads to a decrease in bone mass, destruction of bone microstructure, reduction of bone strength, and a type of systemic bone metabolism disease prone to fracture ${ }^{[2]}$. In recent years, it has been recognized that PMOP is caused by aging and estrogen deficiency. As the basic pathogenesis of aging, oxidative stress plays an important role in the occurrence of related diseases. Estrogen is a powerful antioxidant, and increased accumulation of reactive oxygen species induces oxidative stress, eventually lead to PMOP ${ }^{[3]}$. It is generally believed that with the increase of reactive oxygen species (ROS) and oxidative damages, the formation and survival of bone osteoblasts decrease, while osteoclast differentiation and activity increase ${ }^{[4,5]}$. Some researches show that acupuncture which is on the basis of conventional oral calcium improves bone density and serum estradiol levels in postmenopausal osteoporosis patients. The mechanism may be that acupuncture can increase the estrogen level and promote Calcium, can affect the bone turnover in order to increase bone density ${ }^{[6]}$. Oxidative stressmediated balance of the FoxO- $\beta$-Catenin-PPARy signal axis may play an important role in bone marrow mesenchymal stem cells (BMSCs), $\beta$-Catenin plays a cental pole on the regulation of osteogenic differentiation and osteoblast activity in the bone marrow fatty environment ${ }^{[7]}$.

At present, the prevention and treatment of osteoporosis (POP) mainly depends on chemical drugs, but there are certain side effects ${ }^{[8]}$, and the long-term effect is unsatisfactory. Estrogen therapy, as a complementary replacement therapy for PMOP, has a long-term application with definite effects, but it is easy to cause adverse events such as breast cancer and endometrial cancer ${ }^{[9]}$. The side effects of other medicines which are commonly used in clinical practice have also become increasingly prominent. Adverse reactions such as atypical femoral fractures and mandibular necrosis caused by the use of bipionate have also attracted more and more attention ${ }^{[10]}$. The findings have affected the acceptance of many patients. Therefore, it is significant to find a safe, effective, green, natural, and non-side effect treatment. And the first-line clinical medicine is mostly focused on inhibiting abnormally active bone resorption dominated by osteoclasts. Simply inhibiting osteolysis is difficult to fundamentally treat osteoporosis. The mature growth of osteoblasts is the basis of bone health, so we should pay more attention to the research on methods of promoting bone formation.

Acupuncture therapy is a important therapy for the prevention and treatment of chronic metabolic diseases in Ttraditional Chinese medicine. The clinical treatment of acupuncture for this disease is mainly to strengthen the kidney and bones, while regulating the spleen and kidney. The clinical effect of acupuncture on this disease is definite, and it can significantly improve the symptoms of low back pain, limb weakness and kidney deficiency caused by postmenopausal osteoporosis. Acupuncture can not only alleviate and improve clinical symptoms such as pain in patients with osteoporosis, but also can prevent osteoporosis by regulating the level of endocrine hormones in the body, increasing bone density, and improving abnormal bone metabolism ${ }^{[11,12]}$.

The currently recognized principles of traditional Chinese medicine treatment of osteoporosis are syndrome differentiation, overall regulation,prevention and treatment ${ }^{[13]}$. "Overall adjustment acupuncture"is a kind of acupuncture treatment based on traditional Chinese medicine,which is a effective, 
systematic, comprehensive and multi-pathway treatment of osteoporosis. The "overall adjustment acupuncture" is based on more than 20 years of clinical experience and experimental verification. It combines the main etiology and pathogenesis of osteoporosis with kidney and spleen deficiency. It is involving a systematic process for osteoporosis. It combines acupuncture, moxibustion, and skin needle organically to stimulate the meridian system such as the skin, collaterals, and meridians,according to the full regulation of this therapy, osteoporosis has been treated by improving clinical symptoms and patients' quality of life through multiple channels ${ }^{[14]}$. During the early stage of clinical treatment, it was found that the "overall adjustment acupuncture" method has a good effect on postmenopausal osteoporosis, but a systematic and comprehensive clinical study has not been conducted, and its mechanism is not clear. This topic is to study the efficacy, safety and mechanism of acupuncture therapy on PMOP, there are few reports on the treatment of PMOP by sham acupuncture as a control group. Our topic hypothesis is that "overall regulation" acupuncture treats PMOP by increasing estrogen levels of postmenopausal women and improving the imbalance of osteogenic and adipogenic differentiation of BMSCs caused by oxidative stress in human body.

\section{Methods/design}

\section{Study design}

This is a randomized, sham-controlled, patient and assessor-blinded trial. A target sample of 104 participants will be recruited from the acupuncture clinic at the first affliated hospital of Dali university and Yunnan Provincial hospital of Traditional Chinese Medicine. The present protocol followed the SPIRIT guidelines and fulfilled the SPIRIT checklist(Additional file 1). The flow chart is shown in Fig.1. The protocol is in line with the principles of the Declaration of Helsinki and has been approved by Institution review board (IRB) of Yunnan Provincial hospital and Traditional Chinese Medicine (approval no.2018-00301). This trial was registered at the Chinese Clinical Trial Registry (ChiCTR1800017581). Any changes which need to be made in the trial protocol will be communicated to all researchers, the ethics committees, and the trial registry. Written informed consent will be signed by each participant.

\section{Sample size, randomization, and blinding}

According to the preliminary research ${ }^{[15]}$, we hypothesize that the effective rate after $\mathrm{OA}$ is $77.8 \%$ and the effective rate after $S A$ is $50 \%$. The calculation equation we used was $a=0.05, \beta=0.20$ and we determined that a sample size of 42 patients in each group would be sufficient to detect the statistical difference between the two groups, allowing for a $20 \%$ withdrawal rate. We will plan to enroll a total of 104 participants with 52 participants in each group. According to the random number sequence generated by computer, the patients will be randomly divided into either the OA group or the SA group in a 1:1 ratio. In order to minimize potential sources of bias ,participants, doctor, outcome assessors, and data analysts will be blinded to the treatment allocation. Due to the special nature of acupuncture, acupuncturists can not be blinded to the treatment.

\section{Inclusion criteria}


Participants who meet all the following requirements will be allowed for enrollment:

1. Women aged $45-70$ years.

2. Patients who meet the diagnostic criteria for PMOP:

\Pain occurs mostly with the hips, lower back and ribs, and slowly onset;

QBrittle fracture occurs under the action of slight external force;

$\triangle T$ There is obvious tenderness point on the lower back or there is a large area of tenderness;

\Scoliosis, kyphosis and other deformities;

QDXA(dualenergy X-ray absorptionmetry) is used to detect femoral neck bone mineral density lower than

-2.5. Severe osteoporosis is accompanied by two or more fractures;

『lmaging examination showed obvious osteoporosis or fragility fracture;

\non-secondary osteoporosis

3. Meet the " atrophic debility of bones" in Chinese medicine diagnostic criteria;

4. Natural menopause for more than 1 year;

5. Other medications or other related interventions for postmenopausal osteoporosis were not received within 4-6 months;

6. Patients voluntarily, informed consent, and signed informed consent, with good compliance.

\section{Exclusion criteria}

Participants meeting any of the following criteria will be excluded:

1. Except for women with abnormal lumbar anatomy (such as severe scoliosis);

2. Exclude related endocrine disorders (such as diabetes, hyperthyroidism, hypothyroidism, thyroid cysts, etc.) that can cause secondary osteoporosis;

3. After uterine or ovarian ablation surgery;

4. Concomitant with diseases such as rickets, rheumatoid arthritis or other diseases that affect the dynamic balance of bone metabolism;

5. In the past 3 months, he has taken drugs that can interfere with bone metabolism (such as glucocorticoids, calcitonin, estrogen, etc.); 
6. Female patients with severe underlying diseases and mental illness who are unable to cooperate with treatment research programmes;

\section{Drug or alcohol abuse;}

8. Those who had received acupuncture treatment;

9. Participating in other clinical trials.

\section{Interventions}

Both OA and SA groups will receive acupuncture sessions for a total of three months. All the pa- tients will receive herbal medicine twice a day for three months. The same doctor (the professor of acupuncture in Yunnan Provincial hospital and Traditional Chinese Medicine who has 20 years of work experience) will slightly adjust the formula each week depending on the changes of the symptoms, pulse, and tongue coating of the patients (main herbal formula components are shown in Table 1). During the trial, patients will be based on pattern differentiation:(1) spleen-kidney Yang deficiency will be given Yougui Pill;(2) liverkidney Yin deficiency will be given Zuogui Pill;(3)kidney deficiency and blood stasis will be given Bushen Huoxue decoction. Participants will get all herbs from the same hospital and the doctor will instruct them how to decoct.

According to the overall adjustment of acupuncture and moxibustion ${ }^{[15]}$, and taking opinions from Chinese acupuncture experts, the essential acupuncture points selected are as follows: BL11(Dazhu); BL23 (Shenshu); ST36 (Zusanli). The additional individualized acupuncture points will be chosen by the practitioners according to the patterns of identification: (1) kidney Yang deficiency will plus DU04(Mingmen),GB34(Guanyuan); (2)spleen-kidney Yang deficiency will plus DU04(Mingmen), GB34(Guanyuan); (2)liver-kidney Yin deficiency will plus SP06(Sanyinjiao),LU11(Taixi);(3)kidney deficiency and blood stasis will plus SP06(Sanyinjiao),BL17(Geshu)(Table 2). A placebo device ${ }^{[16]}$ will be applied in both groups for better implementation of blindness. The schematic diagram of acupuncture treatment is shown in Fig.2. The acupuncture treatment in both groups will take 30 min per session, twice per week. Three months is a course of treatment, a total of one course .

\section{Overall adjustment acupuncture(OA) group}

Acupuncture manipulation will be applied to the acupoints of the body until the patient achieves Qi, including soreness, soreness and distension, pain, numbness, tingling and even comfort. Huatuo brand needles $(0.25 \mathrm{~mm} \times 40 \mathrm{~mm})$ were manufactured by Suzhou medical device company in Suzhou, Jiangsu province, China. After the patient achieves Qi in Shenshu and Zusanli, warm acupuncture will be used, fix the $2 \mathrm{~cm}$-long moxa-stick on the handle of the needle and light it at the root of the needle for 20 minutes.

The parameters of the skin needle are set as follows: Qixing needle: tapping along the first side line of the urinary bladder meridian in the back; moderate stimulation will be applied for the blood stasis and qi 
stagnation type, until redness of the skin and petechiae is observed in the tapping part; mild stimulation was used for the rest of the syndromes until redness in the skin is observed.

The parameters of the cupping are set as follows: moving cupping: smear the tapping spot evenly with Vaseline and move the cup along the first lateral line of urinary bladder meridian back and forth until red blood stasis appears on the skin, according to the patient's tolerance (feeling comfortable, without an obvious pain). Retaining cupping: Leave the cup for 8 to 10 minutes in Dazhui, Shenshu, and a severely painful area.

\section{Sham acupuncture (SA)group}

The procedure and duration of treatment in the SA group will be identical in the OA group except the needles $(0.25 \times 25 \mathrm{~mm}$, blunt tip, manufactured by Suzhou medical device company in Suzhou, Jiangsu province, China) $\square$ Qixing needles tip wrapped with cotton(show in figure3) are blunt tip and there will be no skin penetration or manual stimulation, at the same time cupping is applied to the skin with a small negative pressure comparing with the OA group(show in figure 4).

\section{Practitioner background}

The $O A$ and SA treatments will be handled by acupuncturists who are registered by the Chinese medicine practitioner qualification certificate and who are specialized in acupuncture with more than three years of clinic experience. They will have studied acupuncture for more than 10 years and graduated from a TCM university. All operators will be trained in the overall adjustment of the needle operation in advance and qualified, and will undergo intensive and customized training for a full understanding of the SA procedure and a sham needle device. The techniques for the entire treatment procedure will be standardized between practitioners.

\section{Outcome measures}

Outcomes will be collected at baseline, the middle of treatment, after treatment, and follow-up six months after the completion of the treatment.The overview of the outcome measurement at the different time points is shown in Table 3.

\section{Primary outcome}

The primary outcome is the bone mineral density(BMD), BMD was measured by a dual energy X-ray absorptiometry (manufacturer: DMS, France; model: CHALLENGER C 313). BMD of the lumbar vertebrae (L1-L4) in the two groups will be measured before and after treatment.

\section{Secondary outcome}

Pain score The pain score will be assessed for the lower back. A Visual Analogue Scale (VAS) will be used to measure each group before and after treatment (see attached Table 4). 
The TCM syndrome scores will be recorded by the same professor once a week to adjust the acupuncture and herbal treatment [17] and evaluate the improvement of the patients' TCM syndromes (see attached Table 5).

Indicators for Quality of life Based on Quality of Life (QOL) and 36-Item Short Form Survey (SF-36), combined with the specificity of osteoporosis and clinical situations, a self-rating scale in line with Chinese ethnicity was developed (see attached Table 6).

\section{Blood test}

(1) Sex hormones The fasting blood samples will be drawing in every month before and after treatment by the nurse. The chemiluminescence immunoassay (CLIA) will be used to determine serum sex hormone levels include estradiol (E2), FSH (Model: Switzerland ELECSYS2011).

(2) Antioxidant index Before and after intervention, $5 \mathrm{cc}$ of venous blood sample were obtained in nonfasting condition. Samples were frozen in $-70^{\circ} \mathrm{C}$. Advanced oxidation protein products (AOPP), and total antioxidant capacity (TAC) (with 2, 2-diphenyl-1-picrylhydrazyl [DPPH] oxidation) were measured with the following methods: Walwadkar [18], Kataaha et al. [19], Girbal et al. [20], Kitajima [21] and Janaszewska et al. [22].

(3) Bone metabolism index The fasting venous blood was collected before treatment, one course of treatment and at the end of treatment respectively. The enzyme-linked immunosorbent assay (ELISA) (biocell enzyme standard instrument) was used to determine BGP and BALP before and after treatment.

(4) Pathway signaling proteins Western blot was used to detect the expression of $\beta$-Catenin, FoxO3a and PPAR y2 protein in bone tissue(Multiskan spectrum,American(Multiskan spectrum)).

Follow-up Six months after the completion of the treatment, follow-up will be performed by telephone to ask if the participants were fracture and the recurrence of pain.

\section{Adverse events}

Safety will be assessed by body temperature,pulse,breathe, blood pressure,renal function test, liver function test. These indicators are detected during the period of screening and after 12-weeks' treatment. We will monitor adverse events for each treatment during the trial, including hematomas and acute pain. Any adverse events or reactions that are thought to be causally associated with the intervention will be recorded, managed, and reported to the study coordinators. Serious adverse reactions will be reported to the ethical committee.

\section{Data analysis}

All collected data results were compiled using SPSS21.0 statistical software package, and collated, checked and statistically analyzed. The comparison between the data groups was performed by $\chi 2$ test, and the measurement data was consistent with the normal distribution and the homogeneity of the 
variance. The mean \pm standard deviation $( \pm S)$ indicates that the rank sum test is used for comparison between grade data sets that do not satisfy the normal distribution and the homogeneity of the variance. $\mathrm{P}<0.05$ was significant for the difference. The variance analysis will be performed for the difference between the two groups and within the group. We will perform a regression analysis to examine the causal relationship between the $\mathrm{E} 2, \mathrm{TAC}$ and $\beta$-Catenin, and the primary outcome.The statisticians who are independent of the research team will conduct data analysis.

\section{Discussion}

Acupuncture therapy is a major component of TCM and is increasingly widely used because it is noninvasive and has fewer side effects ${ }^{[23-25]}$. Drugs for PMOP exists some side effects ${ }^{[8]}$, and the long-term effect is unsatisfactory. Some clinical studies have reported the effect of acupuncture on patients with osteoporosis. While a recent meta analysis reported that the quality of evidence was generally low and most studies had small sample sizes(ranging from 30 to 100),therefore, the evidence for the use of acupuncture in postmenopausal women with osteoporosis is not conclusive, more high-quality, largesample, multi-center, and well-designed randomized controlled trials should be conducted to provide a reliable basis for further confirming the exact efficacy of acupuncture for postmenopausal osteoporosis [26]. Compared with previous studies, we combined the TCM therapy with scientific and rigorous experimental design to explore the effect of OA on women with PMOP. The selection of acupoints and herbs is based on the syndrome differentiation in order to adequately demonstrate the dialectical treatment of TCM.

The evidence for long-term efficacy of acupuncture in patients with PMOP is not sufficient. This trial will conduct a 6 months follow-up of the patient to observe the long-term efficacy of the overall acupuncture treatment for PMOP. However, a neurological study ${ }^{[27]}$ showed that although sham acupuncture and placebo had the same effectiveness as manual acupuncture in terms of reduction of symptoms and objective physiological outcomes, verum acupuncture was superior to sham acupuncture in improving both peripheral and brain neuro-physiological outcomes.

Among menopausal women, bone mineral density decreases while the bone fragility increases,the fracture in lumbar back and femur are always the commonly end results of these patients. The ovarian function of postmenopausal women declines,so estrogen deficiency appears. In this process, lipid peroxidation and oxidative stress state ${ }^{[28]}$ are induced. Oxidative stress can induce the aging of BMSCs in bone marrow, and hinder the differentiation of BMSCs into osteoblasts ${ }^{[29]}$, which leads to osteoporosis. This mechanism includes transcription factor FoxO3a, BMSCs osteogenic differentiation key factor $\beta$-Catenin and lipogenic key factor PPARY ${ }^{[30]}$. Oxidative stress induces the binding of core protein $\beta$-Catenin with FOXO3a and PPAR $y$ in the pathway, which inhibites the osteogenic differentiation of bone marrow and turns to lipogenic differentiation. The oxidative stress induces because of the decrease of estrogen level in patients resulted in the adipogenic differentiation and the imbalance of osteogenic differentiation of BMSCs. Early clinical observation of "overall adjustment acupuncture" can effectively improve the bone density, reduce pain, improve the quality of daily life of osteoporosis patients, and it is same to PMOP patients. Therefore, 
the subject uses "overall adjustment acupuncture" method, and selects bone density, hormone level, antioxidant index, bone metabolism index, pathway index to prove the efficacy, safety and mechanism of "overall adjustment acupuncture" for PMOP. Our hypothesis is that "Overall adjustment acupuncture"can improve the hormone level which plays plays antioxidant role, adjust the imbalance of adipogenic differentiation and osteogenic differentiation of BMSCs, increase the formation of osteoblasts, increase the bone density, reduce the pain of patients, improve the quality of daily life of patients, prevent the occurrence of fractures, and improve the clinical outcome of postmenopausal osteoporosis patients.

\section{Trial status}

This trial was registed at Chinese Clinical Trial Registry, registration date:10 August 2018, registration number:ChiCTR1800017581.At the time of initial manuscript submission, recruitment had already started (1 August 2018), but it has not been completed. The last patient is expected to be included in the study on 1 August 2020. I confirm that this the protocol version number 1.0 is the version registered at the date that I have provided.

\section{Abbreviations}

PMOP:Postmenopausal Osteoporosis, OA:Overall adjustment acupuncture, SA:Sham acupuncture, BMD:bone mineral density, E2:estradiol,FSH:follicle-stimulating hormone, BGP:bone gla protein, BALP:bone alkaline phosphatase, TAC:total antioxidant capacity, DXA:dual-energy X-ray absorptionmetry, AOPP:advanced oxidation protein products, VAS:visual pain scale score, TCM:Traditional Chinese Medicine, QOL:quality of daily life, BMSCs:bone marrow mesenchymal stem cells, SD:standard deviation;

\section{Declarations}

\section{Ethics approval and consent to participate}

The research plan has already been clinical trials of traditional Chinese medicine hospital ethics committee approval in Yunnan province, China (grant no. (2018) (003) - 01). Central ethical approval has been confirmed from ethics committee of Yunnan provincial hospital of traditional Chinese medicine ethics committee (ref approval no. (2018) (003) - 01) and we will not begin recruiting at other centres in the trial

until local ethical approval has been obtained. The research registered on the Chinese clinical trial registry (http://www.chictr.org.cn/, ID: ChiCTR1800017581). Each participant will sign an informed consent form. Patients will be enrolled in the study and will be recruited only once and will not receive any financial compensation for their participation in the study.

\section{Consent for publication}

All subjects participating in the image acquisition signed the consent form and consent for publication.

\section{Availability of data and materials}


The datasets during and/or analysed during the current study available from the corresponding author on reasonable request.

\section{Competing interests}

The authors declare that they have no competing interests.

\section{Funding}

This project is supported by National Natural Science Foundation (NO: 81560799)

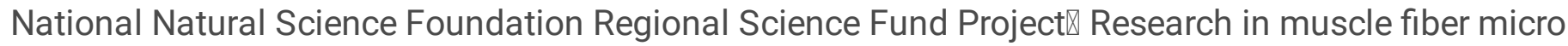
structure and mechanical properties of invigorating spleen and tonifying kidney plus reinforcing bone acupuncture method on osteoporotic hip fracture (Number:81560799). The funding body was not involved in the design of the study and collection, analysis, and interpretation of data and in writing the manuscript.

\section{Authors' contributions}

Conceptualization: Ren ZQ,Zhao R.Data curation:Ren ZQ, Zhao R,Ao GF,Huang M.Formal analysis:Ren ZQ, Zhao R.Funding acquisition:Zhao R.Investigation: Huang M.Methodology: Ren ZQ, Zhao R,Wang YF,Chen HX.Project administration:Zhao R.Resources:Ren ZQ, Zhao R,Ao GF.Software:Zhao HD,Lai MX .Supervision:Zhao R.Validation: Ren ZQ,Zhao R.Visualization: Ren ZQ,Zhao R,Huang M,Wang YF.Ren ZQ drafted the manuscript, and all the authors contributed to writing of the manuscript. All authors read and approved the final version.

\section{Acknowledgements}

Thanks to all the patients, nurses, and professionals in the first affiliated hospital of Dali university and Yunnan Province Hospital of Traditional Chinese medicine who provided valuable input to the trial. We would also like to thank the staff of the Clinical Research Center, the first affiliated hospital of Dali university , for their advice and support throughout the trial process.

\section{Authors' information}

Corresponding author: Zhao R, female, chief physician, professor, eYunnan university of traditional Chinese medicine, Kunming, China. E-mail: kmzhaorong@qq.com. Address: 88 Baita road, Panlong district, Kunming city, Yunnan province, China.

Authors

1)Ren $Z Q$, female,Medical $P h D,{ }^{a}$ Nanjing university of Chinese medicine,Nanjing,China; ${ }^{b}$ The first affiliated hospital of Dali university, Dali, Yunnan Province, China. E-mail:346682574@qq.com(First Author).

2)Wang YF,female,master, 'School of Acupuncture-Tuina and Rehabilitation,Yunnan University of Traditional Chinese Medicine,Kunming,Yunnan Province,China E-mail:1852051064@qq.com. 
3)Ao GF,male,deputy chief physician, ${ }^{b}$ The first affiliated hospital of Dali university, Dali, Yunnan Province, China.E-mail:aoguangfu@163.com.

4)Chen $\mathrm{HX}$,male,master, 'School of Acupuncture-Tuina and Rehabilitation,Yunnan University of Traditional Chinese Medicine,Kunming,Yunnan Province,China.E-mail:1393937032@qq.com.

5)Huang M,female,master, ${ }^{d}$ Department of acupuncture, Kunming Minicipal Hospital of Traditional Chinese Medicine,Kunming,Yunnan Province,China.E-mail:445746826@qq.com.

6)Lai MX,male,master, ' ${ }^{2}$ school of Acupuncture-Tuina and Rehabilitation,Yunnan University of Traditional Chinese Medicine,Kunming,Yunnan Province,China.E-mail:1904409819@qq.com.

7)Zhao HD,male,master, ${ }^{b}$ The first affiliated hospital of Dali university, Dali , Yunnan Province, China.Email:285010162@qq.com.

\section{References}

1. Jing D. The relationship between Calcium intake,physical activity and perimenopausal bone loss[D], Central South University; 2010.

2. Jinyan L, Ying c, Yanli T, Rong Z. Mechanism of the effect of acupuncture treatment of osteoporosis. Chinese Journal of Osteoporosis. 2014;20(03):315-321.

3. Qingyu Y, Chenguang L. Research Progress on Mechanism of Acupuncture for Postmenopausal Osteoporosis. Geriatrics \& Health Care. 2019;25(01):114-117.

4. Genestra M. Oxyl radicals, redox-sensitive signalling cascades and antioxidants. Cellular Signalling. 2007;19(9).

5. Cullinan SB, Diehl JA. PERK-dependent activation of Nrf2 contributes to redox homeostasis and cell survival following endoplasmic reticulum stress. J Biol Chem. 2004;279(19):20108-20117.

6. Xiangling H. Effect of Acupuncture on Bone Mineral Density and Serum Estrogen in Postmenopausal Osteoporosis Patients. Hunan Journal of Traditional Chinese Medicine. 2016;32(11):86-87.

7. Donghao G, Deqiang C, Guoqing T, Zhanwang X. Investigating the development of bone marrow adiposis in primary osteoporosis based on signal crosslinking. Chinese Journal of Osteoporosis. 2019;25(08):1176-1180.

8. Cheng W, Yu C, Yongqing G, Lingqiang Z. Research progress of drugs for osteoporosis. Chinese Science Bulletin. 2014;59(13):1209-1214.

9. Yan F, Tao Z. An overview on the advance in the treatment of postmenopausal osteoporosis. Chinese Journal of Osteoporosis. 2019;25(08):1192-1200.

10. Sundeep K, P BJ, W DD, et al. Benefits and risks of bisphosphonate therapy for osteoporosis. Journal of Clinical Endocrinology and Metabolism. 2012;97(7).

11. Guoping N, Shuxin C, Fei K. Clinical Analysis of Chinese Acupuncture and Massage in Treating for Primary Osteoporosis. Jounnal of Chinese Medicine. 2015;30(10):1527-1529. 
12. Yusheng L. Effect of warm acupuncture on bone mineral density in postmenopausal osteoporosis patients. Asia-Pacific Traditional Medicine. 2015;11(06):81-82.

13. Jirong G, Hongxin Z, Xaioming W, et al. Expert consensus on the prevention and treatment for primary osteoporosis with traditional Chinese medicine (2015). Chinese Journal of Osteoporosis. 2015;21(09):1023-1028.

14. Rong Z, Zili L, Jianming W, Gendong X. Combination of acupuncture with cupping increases life quality of patients of osteoporosis. Chinese Acupuncture and Moxibustion. 2008;28(12):873-875.

15. Chengbin $L$, Jinlong $X$, Zengrong $Y$, Kun H, Rong Z. Clinical study of the treatment of primary osteoporosis with overall regulation of acupuncture. Chinese Journal of Osteoporosis. 2016;22(11):1459-1465.

16. Baoyan L, Huanfang X, Rui M, Qian M, Shiyan Y, Zhishun L. Effect of blinding with a new pragmatic placebo needle: a randomized controlled crossover study. Medicine. 2014;93(27).

17. Zheng YY\Guiding principles of clinical research on new drugsof traditional Chinese Medicine. Bei Jing: China Medical Science and Technology Publishing House区2002: 356-360区

18. X D, B Z, G H. U-shape association between white blood cell count and the risk of diabetes in young Chinese adults. Diabetic medicine: A journal of the British Diabetic Association. 2009;26(10):955-960.

19. Sedigheh M, Jafarian KSR, Farzaneh R, Fereshteh S. Association between diabetes complications and leukocyte counts in Iranian patients. Journal of Inflammation Research. 2012;5.

20. JC C. White Blood Cell Count Is Associated With Macro- and Microvascular Complications in Chinese Patients With Type 2 Diabetes. Diabetes care. 2004;27(1):216-222.

21. Miaoyan Q, Weili S, Xiaomin S, et al. Effects of prediabetes mellitus alone or plus hypertension on subsequent occurrence of cardiovascular disease and diabetes mellitus: Iongitudinal study. Hypertension (Dallas, Tex. : 1979). 2015;65(3).

22. Shim WS, Kim HJ, Kang ES, et al. The association of total and differential white blood cell count with metabolic syndrome in type 2 diabetic patients. Diabetes Research and Clinical Practice. 2006;73(3).

23. Tan L, Tong Y, Sze SCW, et al. Chinese herbal medicine for infertility with anovulation: a systematic review. J Altern Complement Med. 2012;18(12):1087-1100.

24. Ried K, Stuart K. Efficacy of Traditional Chinese Herbal Medicine in the management of female infertility: A systematic review. Complementary Therapies in Medicine. 2011;19(6).

25. Liao W-T, Chiang J-H, Li C-J, Lee M-T, Su C-C, Yen H-R. Investigation on the Use of Traditional Chinese Medicine for Polycystic Ovary Syndrome in a Nationwide Prescription Database in Taiwan. Journal of Clinical Medicine. 2018;7(7):179.

26. Kaili W, Yili Z, Yanming X, Xu W, Junjie J, Yifang A. Systemic review and meta-analysis of acupuncture in the treatment of postmenopausal osteoporosis. Chinese Journal of Osteoporosis.. 2019;25(04):452460.

27. Maeda Y, Kim H, Kettner N, et al. Rewiring the primary somatosensory cortex in carpal tunnel syndrome with acupuncture. Deutsche Zeitschrift für Akupunktur. 2017;60(3). 
28. Almeida M, Ambrogini E, Han L, Manolagas SC, Jilka RL. Increased lipid oxidation causes oxidative stress, increased peroxisome proliferator-activated receptor-gamma expression, and diminished proosteogenic Wnt signaling in the skeleton. J Biol Chem. 2009;284(40):27438-27448.

29. Kim J-S, Kim E-J, Kim H-J, Yang J-Y, Hwang G-S, Kim C-W. Proteomic and metabolomic analysis of H2O2-induced premature senescent human mesenchymal stem cells. Experimental Gerontology. 2011;46(6).

30. Silva-Fernández L, Rosario MP, Martínez-López JA, Carmona L, Loza E. Denosumab for the treatment of osteoporosis: A systematic literature review. Reumatologia Clinica. 2013;9(1).

\section{Tables}

\begin{tabular}{|c|c|c|c|c|c|c|c|c|}
\hline & & \multicolumn{7}{|c|}{ STUDY PERIOD } \\
\hline & \multirow[b]{2}{*}{$\begin{array}{c}\text { Time point } \\
\text { (month) }\end{array}$} & \multirow{2}{*}{$\begin{array}{l}\text { Enrollment } \\
-1\end{array}$} & \multirow{2}{*}{$\begin{array}{l}\text { Allocation } \\
0\end{array}$} & \multicolumn{3}{|c|}{ Post-allocation } & \multirow[t]{2}{*}{ Close out } & \multirow{2}{*}{$\begin{array}{l}\text { Follow-up } \\
6\end{array}$} \\
\hline & & & & 1 & 2 & 3 & & \\
\hline \multirow[t]{4}{*}{ PREPARATION } & Enrollment & & & & & & & \\
\hline & Informed consent & $\bar{V}$ & & & & & & \\
\hline & Eligibility screen & $\sqrt{ }$ & & & & & & \\
\hline & Allocation & & $\sqrt{ }$ & & & & & \\
\hline \multirow[t]{2}{*}{ INTERVENTION } & $\mathrm{OA}$ & & & $\sqrt{ }$ & $\overline{\sqrt{ }}$ & $\sqrt{ }$ & & \\
\hline & SA & & & $\sqrt{ }$ & $\sqrt{ }$ & $\sqrt{ }$ & & \\
\hline \multirow[t]{13}{*}{ ASSESSMENT } & $\begin{array}{l}\text { Liver function } \\
\text { kidney function }\end{array}$ & $\sqrt{ }$ & & & & & & \\
\hline & $\mathrm{T}$; BP; P; R & $\sqrt{ }$ & & $\sqrt{ }$ & $\sqrt{ }$ & $\sqrt{ }$ & & \\
\hline & BMD & $\sqrt{ }$ & & & & & & $\sqrt{ }$ \\
\hline & E2; FSH & $\sqrt{ }$ & & & & & & \\
\hline & BGP; BALP & $\sqrt{ }$ & & & & & & \\
\hline & TAC; AOPP & $\sqrt{ }$ & & & & & & \\
\hline & $\begin{array}{l}\text { FoxO3a; } \\
\text { B-catenin } \\
\text { PPARy }\end{array}$ & $\sqrt{ }$ & & & & & & \\
\hline & VAS & $\sqrt{ }$ & & $\sqrt{ }$ & $\sqrt{ }$ & $\sqrt{ }$ & & $\sqrt{ }$ \\
\hline & TCM syndrome & $\sqrt{ }$ & & & $\sqrt{ }$ & & & \\
\hline & QOL & $\sqrt{ }$ & & & $\sqrt{ }$ & & & \\
\hline & Adverse events & & & $\sqrt{ }$ & $\sqrt{ }$ & $\sqrt{ }$ & $\sqrt{ }$ & \\
\hline & Data analysis & & & & & & $\sqrt{ }$ & \\
\hline & Fracture rate & & & & & & & $\overline{\sqrt{ }}$ \\
\hline
\end{tabular}

Table 1 Main herbal formula selection 


\begin{tabular}{llll}
\hline Pattern & Formula & Composition & \\
\hline $\begin{array}{l}\text { Spleen- } \\
\text { kidney Yang } \\
\text { deficiency }\end{array}$ & Yougui & $\begin{array}{l}\text { Radix Rehmanniae Preparata,Cortex Cinnamomi,Cornu Cervi Degelatinatum,Rhizoma } \\
\text { Dioscoreae,Fructus Corni,Fructus Lyci,Radix Angelicae Sinensis,Cortex Eucommiae,Semen } \\
\text { Cuscutae,Radix Morindae officinalis,Rhizoma Drynaria,R hizoma Sparganii }\end{array}$ \\
$\begin{array}{l}\text { Spleen- } \\
\text { kidney Yin } \\
\text { deficiency } \\
\text { kidney } \\
\begin{array}{l}\text { deficiency } \\
\text { and blood } \\
\text { stasis }\end{array}\end{array}$ & $\begin{array}{l}\text { Zuogui } \\
\text { Rushen }\end{array}$ & $\begin{array}{l}\text { Radix Rehmanniae Preparata, Rhizoma Dioscoreae, Fructus Lycii, Fructus Corni,Radix Achyranthis } \\
\text { Bidentatae, Deerhorn Glue, Deerhorn Glue, Radix Morindae officinalis }\end{array}$ \\
\hline
\end{tabular}

Table 2 Acupuncture point selection

\begin{tabular}{|c|c|}
\hline Point & Location \\
\hline BL11(Dazhu) & $\begin{array}{l}\text { In the spinal region, under the spinous process of the first thoracic spine, } 1.5 \text { cun lateral to the posterior } \\
\text { midline }\end{array}$ \\
\hline BL23(Shenshu) & 1.5 cun beside the spinous process of the second lumbar spine \\
\hline ST36(Zusanli) & At the anterior aspect of the leg 3 cun inferior to ST35 (Dubi) on the line connecting ST35(Dubi) to ST41 (Jiexi) \\
\hline DU04(Mingmen) & Between the spinous processes of the second and third lumbar vertebrae \\
\hline GB34(Guanyuan) & On the lower abdomen at the anterior midline, 3 cun below the umbilicus \\
\hline SP06(Sanyinjiao) & $\begin{array}{l}\text { On the tibial aspect of the leg posterior to the medial border of the tibia, } 3 \text { cunsuperior to the } \\
\text { prominence of the medial malleolus }\end{array}$ \\
\hline LU11(Taixi) & $\begin{array}{l}\text { On the posteromedial aspect of the ankle in the depression between the prominence of the medial } \\
\text { malleolus and the calcaneal tendon }\end{array}$ \\
\hline BL17(Geshu) & Under the spinous process of the seventh thoracic spine on the back, 1.5 cun beside the midline. \\
\hline
\end{tabular}

Table 3 Overview of study visits 


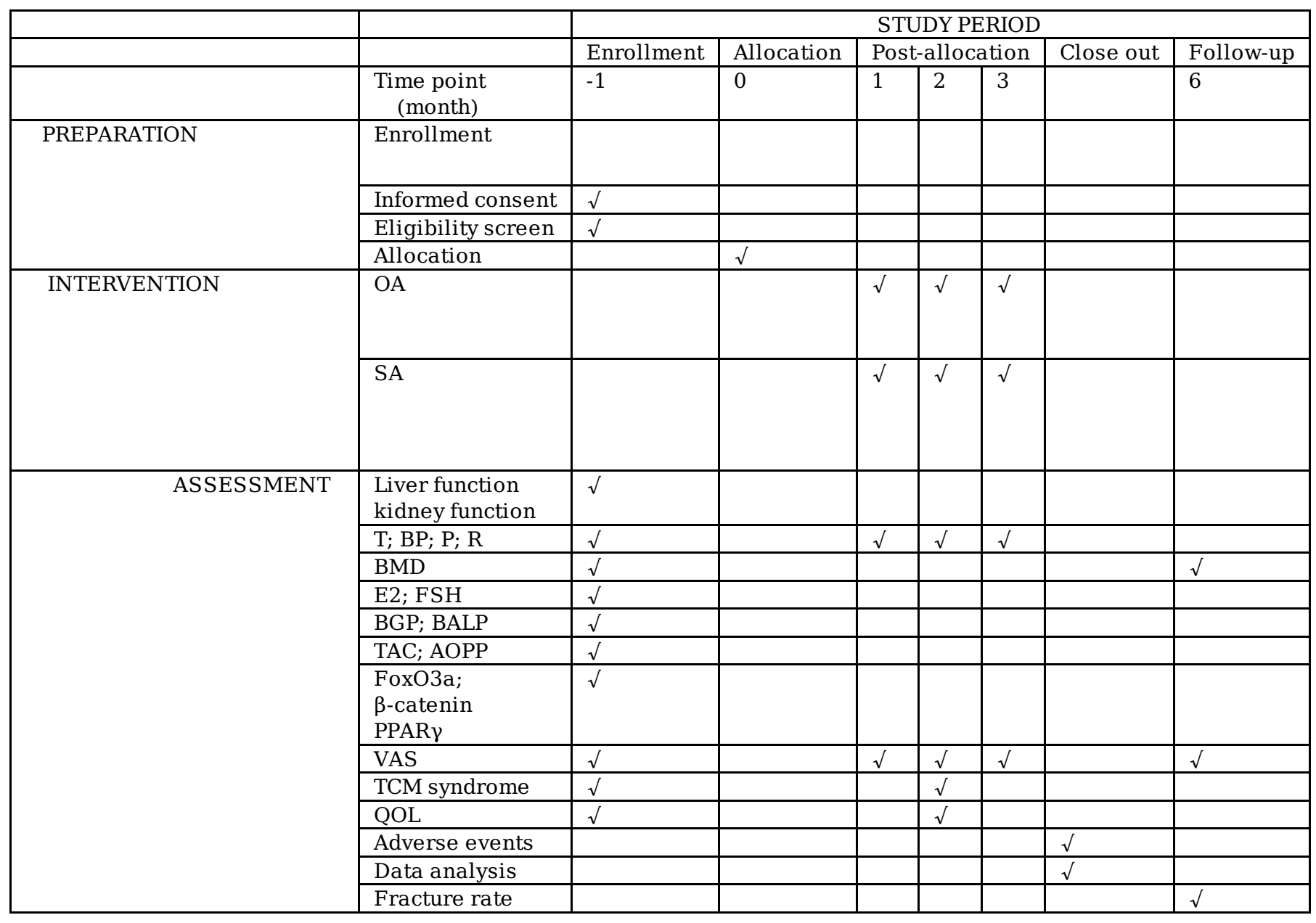

$\mathrm{T}$ : body temperature,BP: blood pressure,P: pulse rate,R: resperation. 
Table 4 Visual Analogue Scale

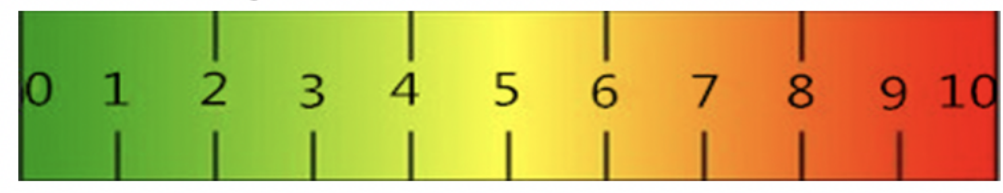

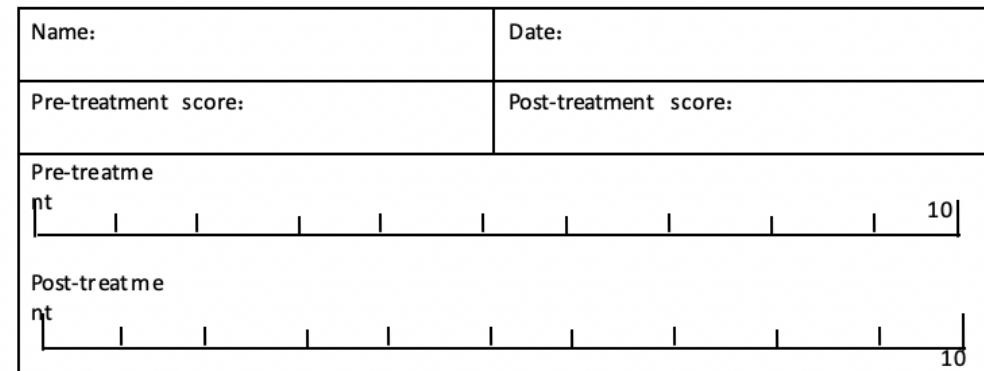

Note: The visual analog scale is a line $10 \mathrm{~cm}$ long with a scale of $0-10$, in which 0 denotes no pain and 10 denotes the worst pain. Patients are asked to mark their pains on the line, and the examiner scores the VAS by measuring the distance in centimeters $(0$ to 10) from 0 to the mark.

Table 5 Osteoporosis symptom grading scale[points $\square$ 


\begin{tabular}{|c|c|c|c|c|}
\hline symptoms & $\begin{array}{l}\text { None } \\
\square 0 \\
\text { pointl }\end{array}$ & $\begin{array}{c}\text { Mild } \\
\square 2 \text { points } \square\end{array}$ & $\begin{array}{l}\text { Moderate } \\
\text { प4points } \square\end{array}$ & $\begin{array}{l}\text { Severe } \\
\square 6 \text { points } \square\end{array}$ \\
\hline $\begin{array}{l}\text { Low back } \\
\text { pain }\end{array}$ & None & 1-3 degrees & 4-6 degrees & 7-9 degrees \\
\hline $\begin{array}{l}\text { Soreness and } \\
\text { wea kness of } \\
\text { waist and } \\
\text { knees }\end{array}$ & None & $\begin{array}{l}\text { After overwalking, I feel a little sore } \\
\text { in my waist and knees. }\end{array}$ & Somewhere in between. & $\begin{array}{l}\text { Soreness and wea kness of } \\
\text { waist and knees continue to } \\
\text { occurland I don't want to } \\
\text { stand and } \\
\text { walk. }\end{array}$ \\
\hline $\begin{array}{l}\text { Lower limb } \\
\text { pain }\end{array}$ & None & 1-3 degrees & 4-6 degrees & 7-9 degrees \\
\hline $\begin{array}{l}\text { Impotence } \\
\text { and } \\
\text { weakness in } \\
\text { the lower } \\
\text { limbs }\end{array}$ & None & $\begin{array}{l}\text { after walking } \square \geq 1 \mathrm{~km} \rrbracket \mathrm{I} \text { may have sore } \\
\text { lower limbs } \\
\text { occasionally. }\end{array}$ & $\begin{array}{l}\text { after walking } \\
\square 300 \mathrm{~m}-1 \mathrm{~km} \square \mathrm{I} \text { may feel weak } \\
\text { and sore in my lower limbs. }\end{array}$ & $\begin{array}{l}\text { after standing and walking } \square \\
300 \mathrm{~m} \square \mathrm{My} \text { legs may } \\
\text { immediately } \\
\text { feel tired. }\end{array}$ \\
\hline $\begin{array}{l}\text { Difficulty in } \\
\text { walking }\end{array}$ & None & $\begin{array}{l}\text { It is inconvenient for me to walk } \\
\text { occasionally, and I do } \\
\text { not feel discomfort walking } \\
\text { less than } \\
100 \mathrm{~m} \text {. }\end{array}$ & $\begin{array}{l}\text { It is difficult for me to walk } \\
\text { even for a short distance } \\
(10-100 \mathrm{~m}) \text {. }\end{array}$ & $\begin{array}{l}\text { It is Difficult for me to stand } \\
\text { and walk. And I cannot walk } \\
\text { for more than } 10 \mathrm{~m} \text {. }\end{array}$ \\
\hline Dizziness & None & $\begin{array}{l}\text { I get dizzy occasionally, but dizziness } \\
\text { does not affect my daily life. }\end{array}$ & $\begin{array}{l}\text { I get dizzy sometimes, and } \\
\text { dizziness would get worse } \\
\text { when I am tired, which } \\
\text { affects my daily } \\
\text { life. }\end{array}$ & $\begin{array}{l}\text { I get dizzy when I move, and } \\
\text { sometimes even fall down, } \\
\text { which seriously affects my } \\
\text { daily life. }\end{array}$ \\
\hline \multicolumn{5}{|l|}{$\begin{array}{l}\text { Pre-treatment } \\
\text { score }\end{array}$} \\
\hline $\begin{array}{l}\text { Post- } \\
\text { treatment } \\
\text { score }\end{array}$ & & & & \\
\hline
\end{tabular}

Note: Osteoporosis symptom grading scale is a measure of pain intensity, using none, mild, moderate, and severe categories with specific scores. Specifically, no pain (0 point); mild pain, without affecting one's work or life (2 points); moderate pain, affecting one's work but not life (4 points); severe pain, affecting one's work and life (6 points).

Table 6 Quality of Life Questionnaire for Patients with Osteoporosis 


\begin{tabular}{|c|c|c|c|c|}
\hline Items & Option & & & Scores \\
\hline I have Calf or hand cramps. () & yes(3) & no(1) & uncertain(2) & \\
\hline $\begin{array}{l}\text { After knowing I had osteoporosis, I felt } \\
\text { anxious. }\end{array}$ & yes(3) & no(1) & uncertain(2) & \\
\hline I do not know much about osteoporosis. & yes(3) & no(1) & uncertain(2) & \\
\hline I think that osteoporosis is a very serious illness. & yes(3) & no(1) & uncertain(2) & \\
\hline I have had fractures. & yes(3) & no(1) & uncertain(2) & \\
\hline I care about a long time period for the treatment of osteoporosis. & yes(3) & no(1) & uncertain(2) & \\
\hline I am quite worried about fractures. & yes(3) & no(1) & uncertain(2) & \\
\hline I think osteoporosis can be cured. & yes(3) & no(1) & uncertain(2) & \\
\hline $\begin{array}{l}\text { I feel that treatment of osteoporosis will } \\
\text { increase my financial burden. }\end{array}$ & yes(3) & no(1) & uncertain(2) & \\
\hline I may keep thinking about my osteoporosis. & yes(3) & no(1) & uncertain(2) & \\
\hline I may not do much physical exercises because of osteoporosis. & yes(3) & no(1) & uncertain(2) & \\
\hline $\begin{array}{l}\text { I do the housework a little slower, and a } \\
\text { little less than before. }\end{array}$ & yes(3) & no(1) & uncertain(2) & \\
\hline I may force me to change my eating habits because of osteoporosis. & yes(3) & no(1) & uncertain(2) & \\
\hline Travel may be restricted because of my osteoporosis. & yes(3) & no(1) & uncertain(2) & \\
\hline I may have less appointments with my relatives, friends, and children. & yes(3) & no(1) & uncertain(2) & \\
\hline I think osteoporosis can have an impact on my life. & yes $(3)$ & no(1) & uncertain(2) & \\
\hline My family may take extra care of me because I have osteoporosis. & yes(3) & no(1) & uncertain(2) & \\
\hline I think I am in poor health. & yes(3) & no(1) & uncertain(2) & \\
\hline After knowing I have osteoporosis, I feel I am old. & yes(3) & no(1) & uncertain(2) & \\
\hline It takes a lot of effort to bend my waist down to do things. & yes(3) & no(1) & uncertain(2) & \\
\hline I often feel pains all over the body. & yes(3) & no(1) & uncertain(2) & \\
\hline I may get upset because of pains. & yes(3) & no(1) & uncertain(2) & \\
\hline I seem to have a kyphosis. & yes(3) & no(1) & uncertain(2) & \\
\hline I am aware that I am getting shorter. & yes(3) & no(1) & uncertain(2) & \\
\hline I am afraid to go out alone or stay home alone. & yes(3) & no(1) & uncertain(2) & \\
\hline The pain caused by osteoporosis interferes with my sleep. & yes(3) & no(1) & uncertain(2) & \\
\hline
\end{tabular}




\begin{tabular}{|c|c|c|c|}
\hline $\begin{array}{l}\text { Sometimes I have a loose tooth or a fallen } \\
\text { tooth. }\end{array}$ & yes(3) & no(1) & uncertain(2) \\
\hline I have tinnitus or hearing loss. & yes(3) & no(1) & uncertain(2) \\
\hline I have memory loss. & yes(3) & no(1) & uncertain(2) \\
\hline I have hyperostosis, herniated discs, or spinal deformities. & yes(3) & no(1) & uncertain(2) \\
\hline Pre-treatment score & \multicolumn{3}{|c|}{ Post-treatment score } \\
\hline
\end{tabular}

Note: The scale is answered by the patient without the physician be involved. The total score of 54 points or less is defined as a normal quality of life, $>54-70$ points as decreased quality of life, 71-80 points as the quality of life decreased obviously, and $>80$ points as a serious decline in quality of life.

\section{Figures}




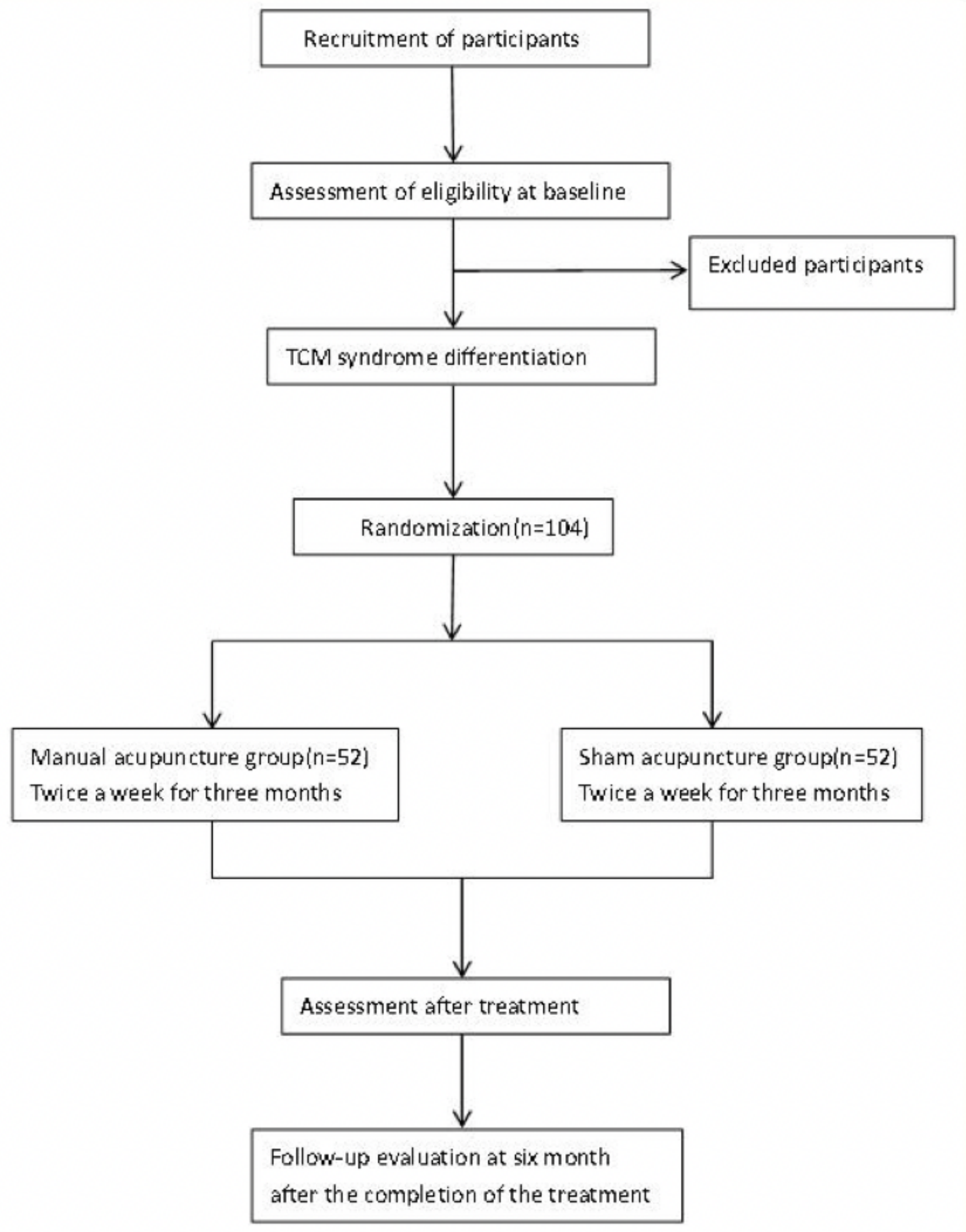

Figure 1

study flow chart 


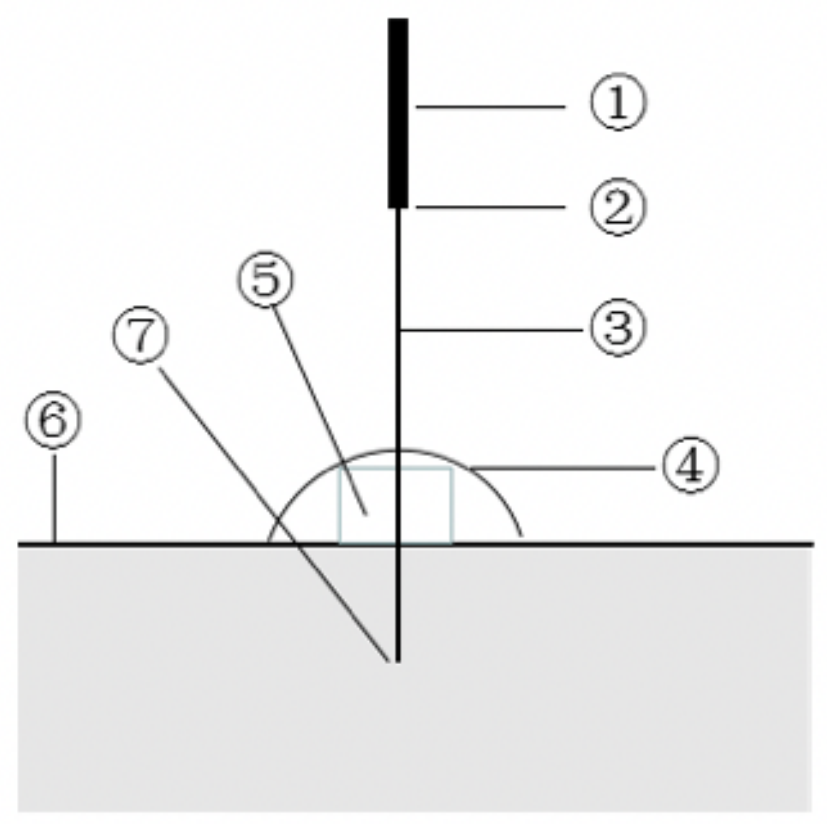

Overall adjustment acupuncture

1.needle handle 2.needle root 3 .neede body 4.Desensitize d transparent 5.adhe sive pad 6. skin 7. sharp tip

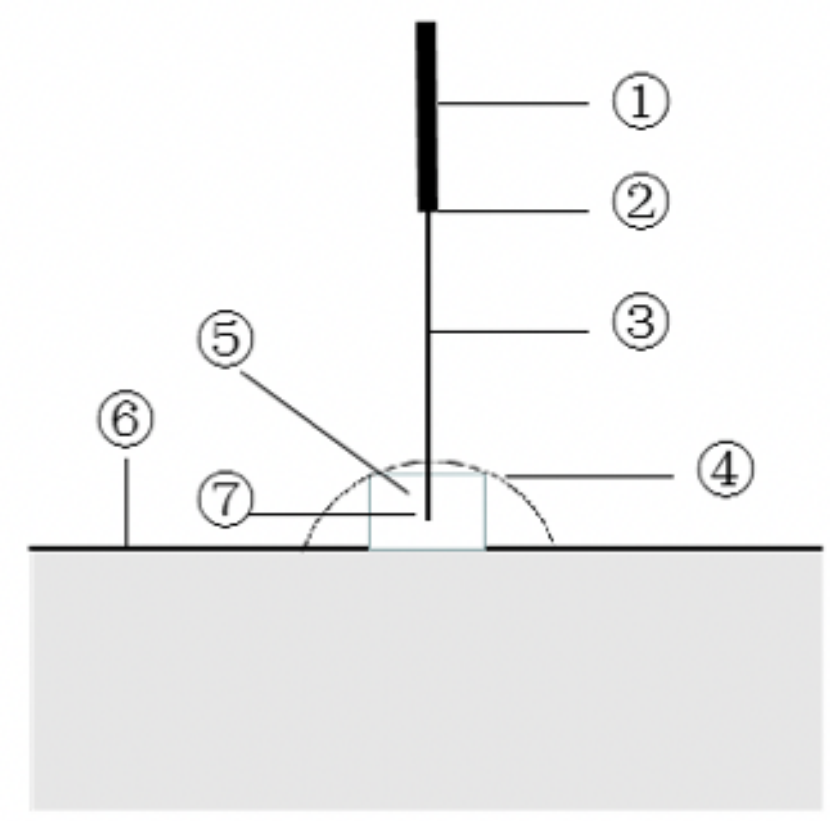

\section{Sham acupuncture}

1. nee dle handle 2.needle root 3 needle body 4.De sensitized transparent 5. adhesive pad 6. skin 7. blunt tip

Figure 2

Schematic diagram of acupuncture device 


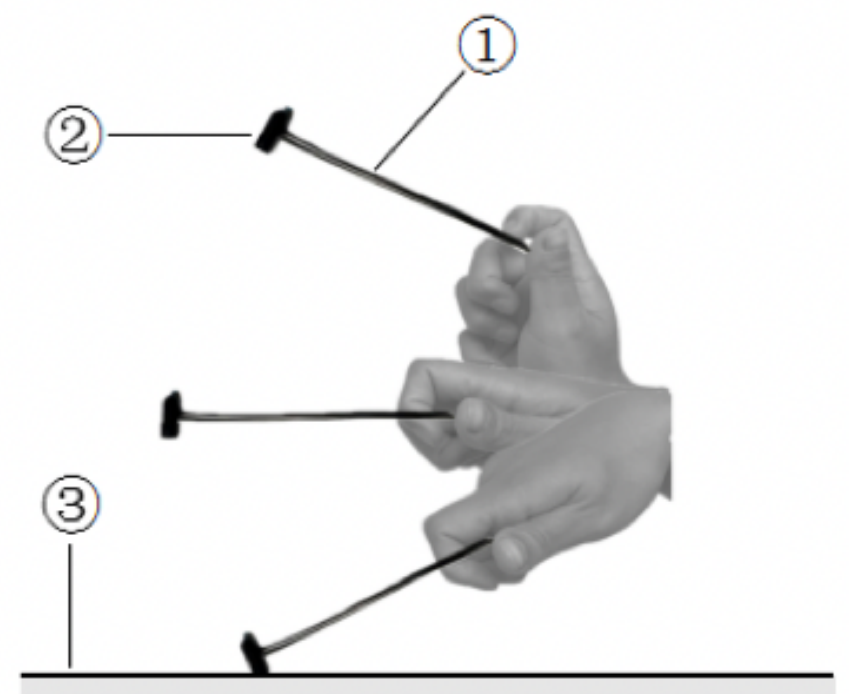

Overall adjustment acupuncture

1. seven-star needle han dle 2.seven-star needle sharp tip 3.skin

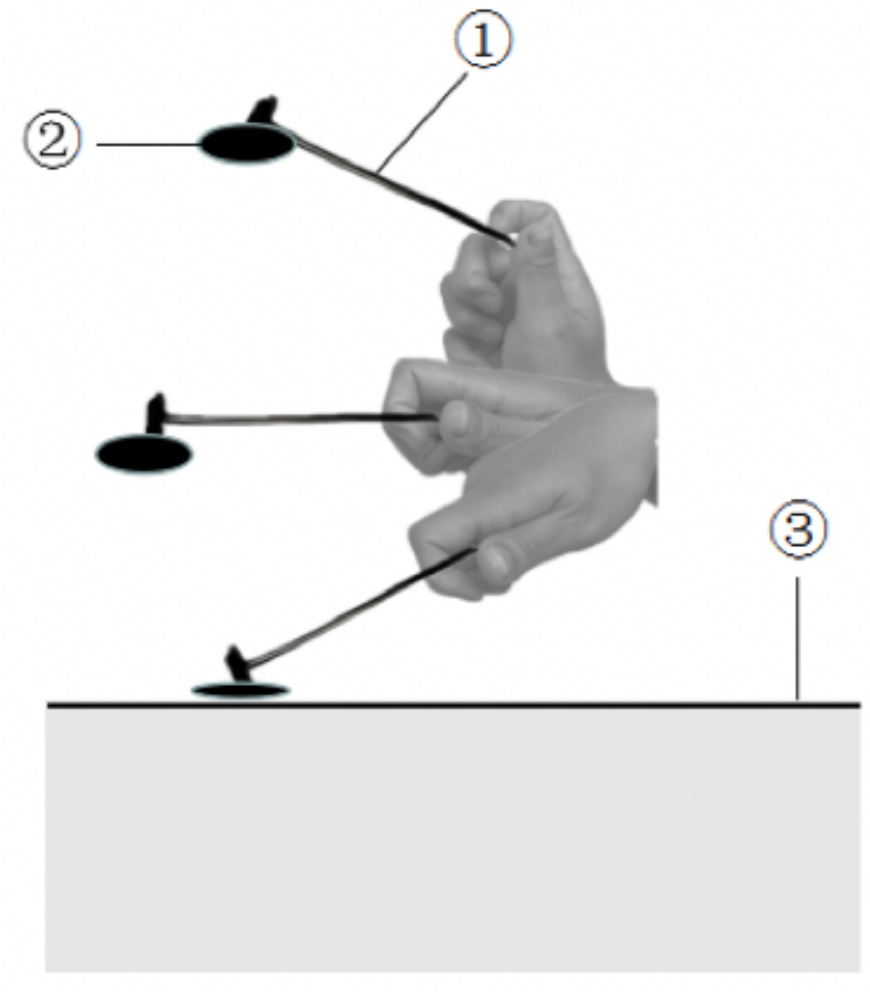

Sham acupuncture

1.Seven-star needle handle 2. seven-star needle blunt tip(needle tip wrapped with cotton 3. skin)

\section{Figure 3}

Schematic diagram of skin needle. NOTE: I confirm the informed consent has been obtained from the individual in figure 3 to publish a potentially identifiable image of them. 


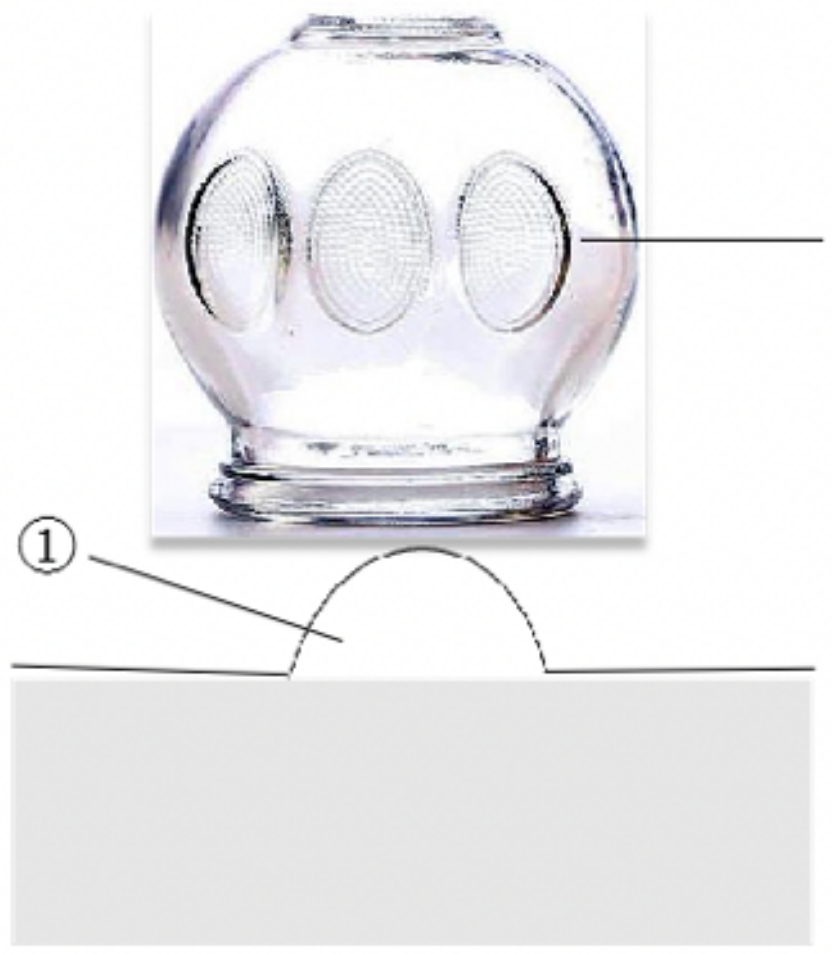

Overall adjustment acupuncture

1.Skin state when cupping 2.cupping

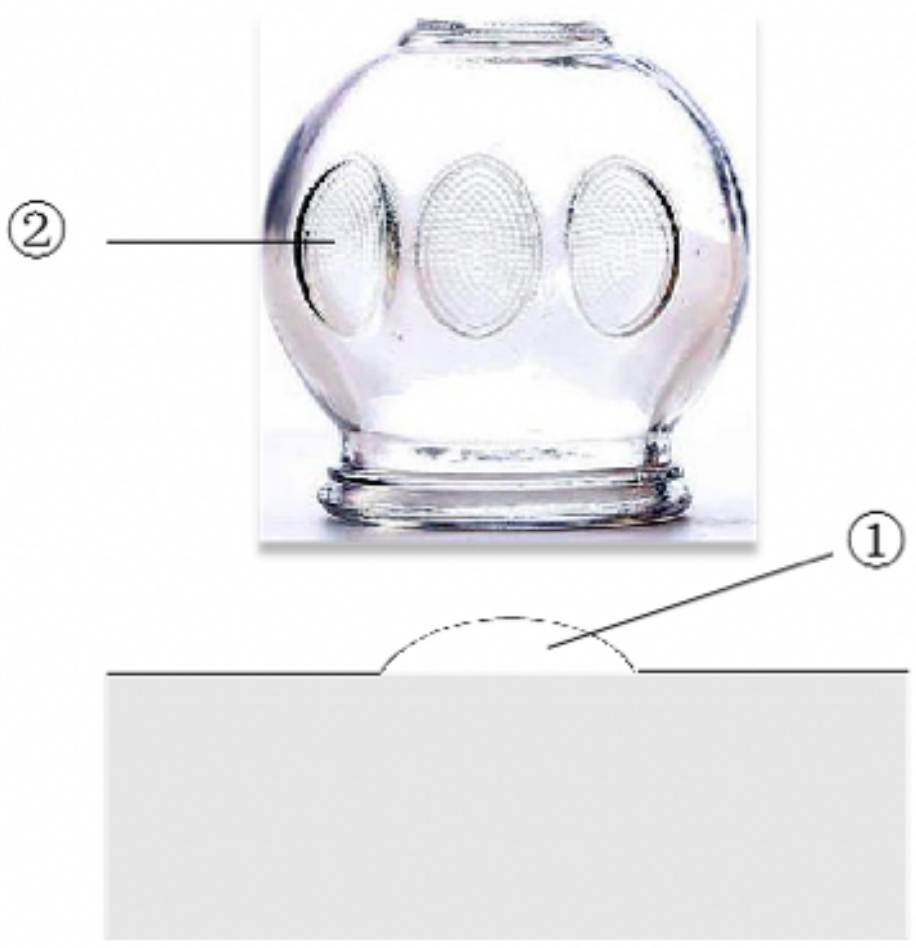

Sham acupuncture

1.Skin state when cupping 2.cupping

Figure 4

Schematic diagram of cupping

\section{Supplementary Files}

This is a list of supplementary files associated with this preprint. Click to download.

- Additionalfile1SPIRITFillablechecklist15Aug2013.doc 